\title{
TENDENCIAS CURRICULARES Y SITUACIONALES DEL NUCLEO DE INGENIERIA DE SISTEMAS, INFORMATICA Y AFINES
}

\author{
José Eucario Parra Castrillón ${ }^{1}$, Carlos Arturo Castro Castro², Mauricio José Amariles Camacho³
}

${ }^{1}$ Magister en Software Libre. Docente investigador UNISABANETA. Grupo de Investigación en Modelamiento y Simulación Computacional GIMSC. Medellín-Colombia. eucarioparra5@gmail.com ${ }_{2}^{2}$ Magister en Geoinformática. Docente Investigador Universidad de San Buenaventura. Medellín-Colombia. carlos.castro@usbmed.edu.co

${ }^{3}$ Magister en Redes y Seguridad de la Información. Docente investigador Universidad de San Buenaventura Medellín-Colombia.mauricio.amariles@usbmed.edu.co

\section{RESUMEN}

El objetivo de este trabajo es analizar tendencias curriculares y situacionales del núcleo de conocimiento de ingeniería de sistemas, informática y afines, con base en percepciones encontradas tanto en sectores empresariales como académicos. Se utilizó un enfoque metodológico mixto, predominantemente cualitativo, debido a que la complejidad conceptual del currículo determina interpretaciones a partir del discurso de los distintos actores que participan en sus procesos. Los resultados obtenidos sobre denominación de los programas, intereses curriculares, métodos de enseñanza, identidad gnoseológica, preocupaciones de las empresas, estado de la formación, educación virtual y educación técnica y tecnológica, advierten sobre auto-críticas que deben hacerse en las facultades de ingeniería, ya que demuestran distanciamientos e incoherencias entre concepciones internas de los programas académicos y variables exógenas originadas en realidades empresariales y sociales.

Palabras clave: currículo de ingeniería, educación virtual, enseñanza de la ingeniería, ingeniería de sistemas, ingeniería informática.

Recibido: 14 de febrero de $2018 . \quad$ Aceptado: 18 de Mayo de 2018.

Received: February 14th, 2018.

Accepted: May 18th, 2018.

\section{CURRICULAR AND SITUATIONAL TENDENCIES OF THE CORE OF SYSTEMS ENGINEERING, INFORMATICS AND RELATED}

\begin{abstract}
The objective of this work is to analyze curricular and situational tendencies of the knowledge core of systems engineering, informatics and related, based on perceptions found in both business and academic sectors. $A$ mixed methodological approach was used, predominantly qualitative, because the conceptual complexity of the curriculum determines interpretations from the discourse of the different actors involved in their processes. The results obtained on denomination of the programs, curricular interests, teaching methods, gnoseological identity, concerns of the companies, state of the training, virtual education and technical and technological education, warn about self-criticisms that must be made in the faculties of engineering, because they show distances and inconsistencies between internal conceptions of the academic programs and exogenous variables originated in business and social realities.
\end{abstract}

Keywords: engineering curriculum, virtual education, engineering education, systems engineering, computer engineering.

Cómo citar este artículo: J. E. Parra Castrillón, C. A. Castro Castro, M. J. Amariles Camacho, "Tendencias Curriculares y Situacionales del Núcleo de Ingeniería de Sistemas, Informática y Afines", Revista Politécnica, Año 14, vol. 14, no. 26, pp. 98-112, 2018. https://doi.org/10.33571/rpolitec.v14n26a9 


\section{INTRODUCCIÓN}

La iniciativa de un análisis del estado y tendencias de la ingeniería de sistemas, informática y afines, surgió en el primer semestre 2016 en la Universidad de San Buenaventura, con la participación de investigadores de esa institución, la Universidad de Antioquia y la Universidad Católica Luis Amigo. La motivación inicial del proyecto tuvo su origen en discusiones y reflexiones que han surgido en organismos como ACOFI (Asociación Colombiana de Facultades de Ingeniería) y REDIS (Red de Programas de Ingeniería de Sistemas y Afines), en torno a cuatro temas fundamentales: la deserción temprana y la baja en la demanda de matrículas nuevas en los programas del núcleo de ingeniería de sistemas, informática y afines (NISIA); la diversidad de denominaciones de estos programas; las indeterminaciones aparentes de sus objetos de estudio y las interpretaciones que sobre la calidad de la modalidad virtual y de los niveles técnico profesional y tecnológico, conllevan a desconfianzas en la sociedad. El objetivo del proyecto fue analizar procesos curriculares y situacionales del NISIA. Es de aclarar que el Ministerio de Educación Nacional hace referencia al Núcleo de Ingeniería de Sistemas, Telemática y Afines, pero en el desarrollo del proyecto se optó por una categorización distinta, considerando que la ingeniería telemática tiene menos vigencia en Colombia que la ingeniería informática.

Dentro del desarrollo del proyecto se estableció que el marco referencial para los procesos curriculares de los programas del NISIA, tiene unos escenarios claramente establecidos en términos de las potencialidades en Colombia para la industria de las TIC y por otra parte, desde las condiciones del Ministerio de Educación Nacional para su funcionamiento y calidad. Paradójicamente, a pesar del desarrollo y potencialidades de las TIC, al interior de las universidades se evidencian con respecto a este núcleo de conocimiento, ciertas problemáticas que se han venido tejiendo en el tiempo, sembrándose dudas y espectros reflexivos en la comunidad académica de ingeniería, en torno a problemas como las siguientes:

1) Orígenes y consecuencias de los volúmenes altos de deserción temprana y las dificultades para retención de los estudiantes, debido a circunstancias académicas.
2) Bajo índice en la matrícula de estudiantes nuevos e incremento de la oferta de programas académicos.

3) Falta de consensos entre la industria de las TIC y las instituciones educativas con respecto al objeto de estudio, en cuanto al hacer y al saber de los ingenieros del NISIA $y$ ausencia de criterios unificados y consensos sobre los fines de la formación de los profesionales y sobre el control de la cobertura y la oferta educativa sobre los programas de este núcleo.

4) Dificultades conceptuales con respecto a currículos, denominaciones, planes de estudio y perfiles profesionales y laborales. Asimismo, falta de acuerdos gnoseológicos e interpretaciones disimiles sobre los objetos de estudio y de investigación y sobre el énfasis metodológico de los programas académicos del NISIA.

5) Distanciamiento entre los patrones de evaluación y propósitos que aplican el Estado, la universidad y las empresas sobre los alcances de la formación e investigación en el campo de las TIC.

6) Dificultades para la concepción educativa y los alcances de la formación en los niveles de tecnología y técnica profesional y en la modalidad virtual de educación.

Acorde con lo anterior, los procesos de indagación se enfocaron en la búsqueda de información para esclarecer inquietudes relacionadas con los requerimientos de las empresas en materia de TIC y las estructuras curriculares de los programas ofertados por las instituciones de educación superior. Pensando en tal dirección se plantearon las siguientes preguntas de investigación: ¿Cuáles son las tendencias formativas a nivel profesional en el NISIA? ¿Cuáles son las tendencias industriales y comerciales en las disciplinas relacionadas con la formación profesional en el NISIA? ¿Cómo debe la universidad responder a los requerimientos de la industria TIC, sin dejar de lado la responsabilidad social que le atañe y el complimiento de las funciones sustantivas de docencia, investigación y extensión? ¿Cuáles percepciones y consideraciones formativas se destacan en relación con los niveles de formación técnica profesional y 
tecnológica y con la modalidad virtual de educación?

Como referentes se tuvieron en cuenta definiciones sobre ingeniería que la conciben como las aplicaciones de las ciencias naturales y las matemáticas para el desarrollo de medios, materiales y recursos que contribuyan a la prosperidad de la sociedad [1] y que además, la entienden como disciplina, arte o profesión enfocada en la aplicación del conocimiento científico para el diseño, desarrollo e innovación de máquinas, sistemas, materias 0 estructuras. Además, se ha considerado un objeto de estudio de la ingeniería de sistemas relacionado con la estructura, mantenimiento, sistematización y automatización de la información [2]. Otra definición la entiende como la rama de la ingeniería que se ocupa de investigación y desarrollo de dispositivos y sistemas informáticos [3]. Con respecto a los perfiles de los ingenieros de NISIA, se consideró por su completitud, la siguiente relación [4]: a) Administrador de proyectos de desarrollo e implantación de sistemas de información y aplicaciones informáticas; b) Arquitecto de tecnología informática; c) Desarrollador de software, d) Analista y administrador de base de datos y de inteligencia de negocios e) Experto en soporte a usuarios de informática, f) Probador de aplicaciones; j) Gerente de informática.

Los interrogantes sobre el objeto de estudio de los programas del NISIA no son nuevos, encontrándose que sobre el objeto de estudio se afirmaba en 1997, por un lado, que el tema central es la tecnología informática, mientras que otras exposiciones decían que lo importante eran los sistemas, la información o los sistemas de información. No obstante, a pesar de estas disimilitudes se concluía que debido a los rápidos cambios en el mundo informático, los currículos deben ser flexibles para poder estar actualizados permanentemente, obligándose agilidad en los procesos administrativos de las universidades para que los cambios curriculares puedan tramitarse expeditamente [5]. Asimismo en 2011 exponían como problema la carencia de acuerdos sobre lo que debe ser un ingeniero de sistemas, ya que esa titulación abarca a programadores, técnicos en infraestructura, expertos en planeación de informática y arquitectos de datos. Debido a esto se ha generado un problema de imagen y de posicionamiento de los programas de NISIA, reflejado en aspectos como los siguientes: la demanda de ingenieros no disminuye, pero los salarios siguen decreciendo; las empresas señalan que las universidades no están alineadas con sus necesidades; los empleadores tienen dudas crecientes sobre la utilidad de los profesionales de sistemas y la sociedad, en general, valora cada vez menos el trabajo de estos ingenieros [6]. Se afirma también que en el pasado los ingenieros de sistemas veían su disciplina como el estudio de los conceptos y situaciones relativas a los computadores, pero esa visión fue migrando hacia otra que los concibe como profesionales expertos en la atención de necesidades relativas a la gestión, flujo y procesamiento de la información, utilizando sistemas tecnológicos [7].

El análisis de tendencias en aspectos curriculares, se consideró con base en las diferencias entre los conceptos de plan de estudio y currículo. El primero entendido como la organización de las secuencias de enseñanza y los contenidos de un programa de formación. Mientras que el segundo comprende principios, medios, procesos, criterios, indicadores evaluativos, condiciones socio-culturales, contextos endógenos y exógenos incidentes en la formación, y los contenidos de la enseñanza, entre otros aspectos [8], [9]. Según esto, un plan de estudios de un programa académico, es apenas un componente del currículo.

\section{MATERIALES Y METODO}

En el desarrollo del proyecto se siguió una metodología mixta que integró técnicas de investigación cualitativa, cuantitativa y documental. La investigación cualitativa tuvo un enfoque fenomenológico, con el propósito de comprender las realidades en torno al currículo del NISIA y de interpretar sus contextos y conceptos desde el sentir de los actores [10]. A su vez, con el componente cuantitativo se buscó recolección y análisis de datos para obtener con el uso de la estadística, patrones sobre la percepción en las empresas sobre aspectos curriculares del NISIA [11]. Como fuentes documentales se tuvo en cuenta información de Sistema Nacional de Información de Educación Superior (SNIES) y del Observatorio Laboral para la Educación (OLE). Además, se hicieron análisis sobre publicaciones de la Red de Programas de Ingeniería de Sistemas y Afines (REDIS), sobre relaciones entre universidad y empresa, la formación técnica y tecnológica y los retos profesionales de los 
ingenieros de sistemas [12], [13], [14], [15], [16], [17].

El proceso investigativo se realizó en el siguiente orden:

Momento 1: Indagaciones sobre tendencias curriculares en los programas del NISIA, con el fin de conocer características de procesos curriculares y planes de estudio. Para esto se utilizó una estrategia de análisis de casos específicos, optándose por referentes preferiblemente de programas con acreditación de alta calidad.

Momento 2: Indagaciones sobre educación virtual en el NISIA. El propósito fue interpretar posturas de actores de la educación virtual (egresados, estudiantes y profesores) sobre asuntos referentes a la formación y a la experiencia vivida en la modalidad virtual. Al tenor de lo anterior se realizaron tres grupos focales: el primero con profesores de la Fundación Universitaria Católica del Norte y del SENA. El segundo con egresados de la unidad académica U de @ de la Facultad de Ingeniería de la Universidad de Antioquia, la Universitat Oberta de Catalunya, la Fundación Universitaria Católica del Norte y el Politécnico Gran Colombiano. Un tercer grupo se realizó con estudiantes de la Fundación Universitaria Católica del Norte y estudiantes de Ingeniería de Sistemas de la unidad U de @ de la U de A. Cada grupo focal dialogaron 6 participantes.

Momento 3: Indagaciones sobre la enseñanza en el NISIA. Se hizo una entrevista en profundidad a un profesional con recorrido amplio en docencia, dirección universitaria y en desempeño profesional en empresas privadas. El tema central fue la pedagogía en el ámbito universitario y el rol del docente en su sentido integral.

Momento 4: Indagaciones sobre expectativas del sector empresarial. Se recogió y sistematizó información obtenida de foros realzados con ingenieros representantes de empresas comprometidas con el desarrollo y aplicación de las tecnologías de la información. El tema fundamental de estos encuentros giró alrededor de las expectativas en las empresas acerca de las competencias y perfiles deseados en los ingenieros que recién egresan de las universidades.

Momento 5: Aplicación de una encuesta a ingenieros del sector empresarial, con el fin de conocer respuestas con respecto a los roles que distinguen a técnicos, tecnólogos, ingenieros y expertos, en el desarrollo de software y gestión de la informática y sobre la importancia de las matemáticas en el desempeño profesional en las empresas. Participaron 50 ingenieros y la encuesta se aplicó utilizando las siguientes plataformas: Google Form, Linkedin y Facebook.

Momento 6: Revisión de ofertas profesionales divulgadas en el primer semestre de 2017 para contratar profesionales en el área de informática. Se utilizó para este propósito las plataformas Linkedin y Computrabajo y se logró establecer cuáles son las tendencias acerca de la formación y perfiles que reclaman las empresas para vincular a los ingenieros en el núcleo de sistemas, informática $y$ afines.

Momento 7: Indagaciones sobre expectativas de la docencia. Para el análisis dentro del componente curricular se hicieron entrevistas a decanos, directores de Programa y profesores con el fin de conocer sus posturas con respecto a: denominación de los programas académicos, características del currículo, tendencias del currículo, relaciones con el sector empresarial, asuntos críticos relacionados con la deserción y el ingreso de nuevos estudiantes, criterios con respecto a la innovación y los procesos de investigación y retos sobre el impacto social. Participaron representantes de las siguientes instituciones: Universidad de Antioquia, Tecnológico de Antioquia, Universidad Nacional, Universidad EAFIT, UNISABANETA, Universidad de San Buenaventura, Universidad de Medellín, Politécnico Colombiano Jaime Isaza Cadavid, UNIMINUTO, Universidad Católica Luis Amigo, Fundación Universitaria Católica del Norte, Corporación Universitaria Américana, UNIREMINGTON e Instituto Metropolitano de Educación (instituciones estas del Departamento de Antioquia). Además, se consultaron académicos de las siguientes instituciones: Universidad del Norte (Barranquilla), Politécnico Gran Colombiano (Bogotá), Universidad Incca (Bogotá), Uniagustiniana (Bogotá) y Universidad de San Buenaventura (Bogotá).

Momento 8: Indagaciones sobre las tendencias de la formación en los niveles de técnica profesional y tecnología. Se recogió información documental y testimonios sobre la formación en los niveles de tecnología y técnica profesional. Se indago sobre el 
sistema educativo del SENA, los ciclos propedéuticos, el proyecto Alianza Futuro Digital Medellín y la iniciativa emergente en 2016 sobre el Sistema Nacional de Educación Terciaria.

\section{RESULTADOS}

Con el fin de ubicar el análisis de tendencias en el NISIA, se ha creado un marco referencial consistente en unos procesos de filtros de datos sobre programas activos con registro calificado vigente y sobre número de egresados. Los datos se obtuvieron de sistemas de información del Ministerio de Educación Nacional (SNIES y OLE) entre marzo y abril de 2017.

En Colombia había 7117 programas con registro calificado activo y en Antioquia 1161. De estos, el $7.8 \%$ en Colombia y el $6.2 \%$ en Antioquia son del NISIA. A su vez, del área de arquitectura, ingeniería, urbanismo y afines (AIUA), en Colombia hay 2152 programas y en Antioquia 343. EI NISIA representa el $25.8 \%$ de esta área en Colombia y el $21 \%$ en Antioquia. Es de anotar que estas cifras encierran programas en las tres modalidades: presencial, virtual y a distancia.

Los datos hallados demuestran que la educación superior en Antioquia y Colombia en su gran mayoría se desarrolla en modalidad presencial y que la educación a distancia ha perdido trascendencia en comparación con la educación virtual. Se encontró que en Colombia, el $5.5 \%$ y en Antioquia el $4.8 \%$, de los programas académicos son en modalidad virtual. En el NISIA el porcentaje de la modalidad virtual en Colombia es del $8.4 \%$ y en Antioquia del $11 \%$.

Asimismo, en Colombia el incremento en el número de graduados entre 2001 y 2015 en los programas de NISIA del $182.24 \%$, estando por debajo de los programas de medicina, derecho, contaduría pública y psicología y siendo muy similar al de administración de empresas (181.28\%). Además, en 2001 el número de egresados del NISIA representaba el $28.7 \%$ de AIUA y en el 2015 el porcentaje fue del $19.6 \%$. Esto quiere decir que con respecto a los demás programas del área de ingeniería y arquitectura, el número de egresados ha bajado proporcionalmente.

En 2001 en Antioquia el número de graduados de NISIA era menor al de administración de empresas, medicina, derecho y contaduría pública. En 2015 su número de egresados es menor que los de administración de empresas, derecho y contaduría pública. Mientras que en psicología el número de egresados se incrementó entre 2001 y 2015 en un $333 \%$ y en derecho en un $315.2 \%$, en NISIA fue del $291 \%$. En comparación con el área AIUA, en 2001 los programas del núcleo NISIA representaban un $7.7 \%$, mientras que en 2015 el porcentaje fue del $7.6 \%$. Con relación al total (entre esos dos años), el porcentaje de programas de NISIA es del $10.12 \%$.

Se encontró también que las denominaciones con mayor número de egresados en Colombia son ingeniería de sistemas (66831), ingeniería de sistemas y computación (4593) e ingeniería informática (4372). No deja de ser llamativo que relacionados con NISIA hay 29 programas distintos en el nivel profesional que cuentan con algún número de egresados. Se encontró también que los programas en los niveles de técnica y tecnología con mayor número de egresados son: tecnología en sistemas (21770), tecnología en análisis y desarrollo de sistemas de información (12319), tecnología en sistemas de información (6657), técnica profesional en ingeniería de sistemas (3467) y técnica profesional en sistemas (2766).

En Antioquia las denominaciones de tecnología de sistemas con 4957 egresados, tecnología en sistemas de información (2428) y técnico profesional en programación de sistemas de información (208), son los que más se destacan del NISIA. Se resalta que en Colombia hay 21 programas del NISIA en el nivel de tecnología que cuentan con egresados, mientras que en Antioquia la cifra es de 11. Igualmente, en el nivel de técnica profesional en egresados en Colombia hay 17 programas y en Antioquia hay 7 .

La tabla siguiente muestra la proliferación de programas de NISIA en Colombia: 
Tabla 1

Numero de egresados en Colombia entre 2001 y 2015 en el núcleo básico de ingeniería de sistemas, informática y afines. Nivel de formación profesional

\begin{tabular}{|c|c|c|c|}
\hline Programa & 2001 & 2015 & $\begin{array}{c}\text { Total entre } \\
2001 \text { y } \\
2015\end{array}$ \\
\hline Administración comercial y de sistemas & 0 & 27 & 645 \\
\hline Administración de informática & 23 & 1 & 156 \\
\hline $\begin{array}{l}\text { Administración de sistemas de } \\
\text { información }\end{array}$ & 44 & 2 & 391 \\
\hline $\begin{array}{l}\text { Administración de sistemas } \\
\text { informáticos }\end{array}$ & 0 & 55 & 887 \\
\hline Administración en informática & 0 & 0 & 75 \\
\hline Administración en informática & 27 & 0 & 53 \\
\hline Administración informática & 5 & 4 & 86 \\
\hline Ingeniería de sistemas & 3797 & 5490 & 66831 \\
\hline $\begin{array}{l}\text { Ingeniería de sistemas con énfasis } \\
\text { En administración e informática }\end{array}$ & 29 & 0 & 171 \\
\hline $\begin{array}{l}\text { Ingeniería de sistemas con énfasis en } \\
\text { software }\end{array}$ & 0 & 1 & 131 \\
\hline $\begin{array}{l}\text { Ingeniería de sistemas con énfasis } \\
\text { En telecomunicaciones }\end{array}$ & 85 & 1 & 1504 \\
\hline Ingeniería de sistemas de información & 31 & 0 & 133 \\
\hline Ingeniería de sistemas e informática & 0 & 83 & 606 \\
\hline Ingeniería de sistemas informáticos & 0 & 27 & 72 \\
\hline Ingeniería de sistemas y computación & 177 & 305 & 4593 \\
\hline $\begin{array}{l}\text { Ingeniería de sistemas y } \\
\text { telecomunicaciones }\end{array}$ & 48 & 93 & 1456 \\
\hline Ingeniería de sistemas y telemática & 13 & 0 & 308 \\
\hline Ingeniería de software & 0 & 56 & 298 \\
\hline $\begin{array}{l}\text { Ingeniería de software y } \\
\text { comunicaciones }\end{array}$ & 0 & 0 & 61 \\
\hline Ingeniería en computación & 23 & 0 & 82 \\
\hline Ingeniería en informática & 0 & 15 & 45 \\
\hline Ingeniería en multimedia & 0 & 44 & 425 \\
\hline Ingeniería en software & 0 & 65 & 166 \\
\hline Ingeniería en teleinformática & 0 & 23 & 396 \\
\hline Ingeniería en telemática & 0 & 18 & 550 \\
\hline Ingeniería informática & 20 & 427 & 4372 \\
\hline Ingeniería multimedia & 0 & 13 & 21 \\
\hline Ingeniería telecomunicaciones & 0 & 5 & 23 \\
\hline Ingeniería telemática & 10 & 24 & 344 \\
\hline Total & 4332 & 6779 & 84881 \\
\hline
\end{tabular}

Dentro de la investigación se logró además de lo anterior, información de las distintas fuentes que puede resumirse se la siguiente forma, luego de aplicados los instrumentos siguiendo la ruta metodológica planeada:

\subsection{Organización curricular}

1) Las diferencias con respecto a las denominaciones dentro del NISIA, se observan sobre la base de algunas líneas de asignaturas, pero no sobre la configuración esencial de los planes de estudio. Las organizaciones curriculares obedecen a unos mismos patrones para ingeniería de sistemas, ingeniería informática e ingeniería del software $y$ no se evidencian diferencias fundamentales dentro de sus planes de estudio ni dentro de sus currículos. ¿Es mejor una denominación general, como ingeniería de sistemas o informática? ¿Es mejor una definición especificada con una temática como ingeniería del software? Según las fuentes, las distintas denominaciones no han contribuido positivamente a la disciplina computacional, sino que ha confundido tanto a las personas del común, como a quienes quieren estudiar programas de NISIA. La verdad es que con respecto a las denominaciones son más las incertidumbres que las certezas. Los argumentos encontrados en las entrevistas así lo demuestran. Se afirmó que Ingeniería Informática e Ingeniería de Sistemas hay yuxtaposiciones y sobre posiciones que causan confusión, no solo en el publico común, sino también en los académicos y expertos curriculares.

En la opinión de directores de programa y decanos, los dilemas anteriores sobre la denominación de los programas ha dificultado la matrícula de estudiantes nuevos, aunque hay otros factores influyentes tal vez de mayor intensidad.

Se encontró que las denominaciones predilectas en el NISIA son ingeniería de sistemas e ingeniería informática y empieza a perfilarse la ingeniería del software. En relación con SUS planes de estudio, las diferencias no son significativas y en todos los casos tres perfiles son comunes: ingeniería del software, bases de datos y fundamentos de programación y conocimientos de ciencias básicas.

2) En general, la organización curricular se establece con la siguiente composición: un área de ciencias básicas, un área de formación básica, un área de formación específica o profesional y un área optativa. El área optativa puede tener un componente obligatorio y otro de libre elección. El primero está constituida por una suma de créditos que el estudiante debe cursar para completar su plan de estudios, el otro conjunto no es requisito de graduación. Se busca racionalidad en cuanto al número de créditos de los programas académicos, entre 140 y 150, con la idea de hacer más efectivo el tiempo de estudio de los estudiantes y se observa una tendencia de enlazar el plan del 
pregrado con estudios de postgrado a nivel de especialización.

3) Con respecto a las ciencias básicas, se observan diferencias. Mientras que en unas instituciones, reconocidas socialmente por su calidad e impacto, se continúa con la tradicional enseñanza basada en el rigor temático y la catedra concentrada en el docente, en otras se manifiestan intencionalidades por una pedagogía abierta, centralizada en la actividad de estudiante y con mayor flexibilidad en la evaluación. Se observan también tendencias en cuanto a la intensidad de las ciencias básicas, tanto en el número de créditos como en los contenidos, buscándose fortalecer la siguiente línea: calculo, matemáticas discretas, algebra lineal, estadística, física mecánica y física de campos.

Se encontró que con respecto a la formación básica, es crucial mejorar la fundamentación matemática y la lógica de programación si se quieren fomentar procesos formativos en función de aumentar la creatividad. Al mismo tiempo, en los currículos se deben recoger las prácticas mundiales reconocidas para la gestión y desarrollo de la tecnología. Además, se debe revisar el contenido curricular relacionado con la formación del ser (el ingeniero como persona). Esto porque aparte de sus conocimientos técnicos, los ingenieros deben estar preparados para la convivencia empresarial y social, de manera acertada y en sustentación de su calidad de persona.

Según una encuesta aplicada a ingenieros vinculados con el sector empresarial, el $69.6 \%$ afirmó que el conocimiento y aptitud matemática son condiciones necesarias para mayor calidad en los procesos de desarrollo de software, el $73.9 \%$ dijo que lo son para el análisis e interpretación de datos en experimentación con prototipos en los procesos de desarrollo de software y el $65.2 \%$ dijo que lo son para para mayor eficiencia y eficacia en la solución de problemas de informática en las empresas.

4) La práctica empresarial en la generalidad de las instituciones y el proyecto de grado, algunas veces, se consideran oportunidades curriculares para la contextualización de la formación de los ingenieros. El principio básico es que estas dos estrategias puedan darle significado y aplicación a lo que han sido los aprendizajes en el transcurso de su carrera. Recogidos los conceptos emitidos por los entrevistados, se encontró que las estrategias para conectar los procesos curriculares con el sector empresarial en la Ingeniería de Sistemas, Informática o afines, se resume en las siguientes estrategias: prácticas empresariales que los estudiantes realizan terminando su carrera, participación en iniciativas o programas de intervención tecnológica (externas a la institución), programación de eventos al interior de las instituciones sobre actualización o sobre divulgación de tecnologías, programación de muestras informáticas, encuentros con egresados destacados en las empresas o reuniones con representantes de gremios y del sector productivo para análisis curriculares.

\subsection{Educación virtual}

La modalidad virtual es atractiva porque permite cobertura e inclusión de población que por distintas razones no pueden hacer parte de las universidades tradicionales. Es innegable el crecimiento de la oferta de programas de NISIA en modalidad virtual. Esta es ya una tendencia en la educación superior.

En referencia a la actitud del estudiante dentro de esta modalidad, afirmaron las fuentes que sus actividades llegan hasta donde las guías lo exigen, sin ir más allá del requerimiento preciso. En relación con lo pedagógico, no se encontró claridad sobre como es el aprendizaje en el aula virtual de ciertos procesos complejos, algorítmicos, analíticos y relacionales, que caracterizan a ciertas áreas de los programas de NISIA y que en la educación presencial exigen despliegues didácticos especiales de los profesores. Con respecto al compromiso ético de estudiantes, profesores y directivos, según lo expresaron las fuentes, hay vacíos en las actuaciones, sobre todo cuando se parte de la base de una alta confianza en la subjetividad de cada uno. Quedan dudas con respecto a los controles y la ética para garantizar autenticidad, validación y verificación dentro de los procesos formativos y evaluativos.

\subsection{Enseñanza y rol de los docentes}

Una tendencia encontrada, es la búsqueda de alternativas para la enseñanza de la ingeniería, de manera que los aprendizajes sean más significativos para los estudiantes. 
A pesar que el NISIA se caracteriza por su enfoque explícitamente técnico, tecnológico y empresarial, e igualmente, aun con sus propósitos curriculares para el modelamiento matemático, en la docencia se observa preocupación por el factor humano. Las concepciones curriculares apuntan a formar profesionales no solamente con competencias específicas para la informática y los sistemas de información, sino que se plantea la necesidad de educar para la convivencia, la alteridad, el respeto y en general, para todo aquello que se relacione con la dignidad del ser como tal y con la fibra social que se represente en los distintos contextos.

Se encontró que unas preocupaciones continuas en los programas de NISIA, son ¿Que enseñar? y ¿Cómo enseñar?, de manera que se alcancen altas motivaciones en los estudiantes y aprendizajes verdaderamente significativos. Junto con estos interrogantes se encontraron dos tendencias didácticas: el Aprendizaje Basado en Problemas (ABP) y el Aprendizaje Basado en Proyectos. Estas dos estrategias son representativas del aprendizaje significativo, que promulga anclajes entre los conocimientos previos del estudiante y la nueva información que reciben. Sobre el ABP se han encontrado evidencias sobre su aplicación que son exitosas debido a las motivaciones y los retos significativos de las situaciones problemáticas que se utilizan en las clases [18], [19], [20], [21].

\subsection{Formación técnica y tecnológica e interés empresariales}

Se evidenció en los grupos focales y entrevistas que una inquietud sobresaliente que surge en los círculos académicos y empresariales es la relacionada con la finalidad de la formación técnica y tecnológica, ya que desde unos puntos de vista, especialmente académicos, se le asocia exclusivamente con perfiles operacionales de menor escala, e incluso a veces hasta se llega a estigmatizaciones, Mientras tanto en las empresas se le da relevancia a estos niveles de formación, ya que sus egresados resultan esenciales para las funciones productivas.

Una tendencia es el interés por el fortalecimiento de la formación técnica y tecnológica en Colombia, pero no en cuanto a su masificación o al encadenamiento de niveles o ciclos, sino en relación con su reconocimiento, dignificación, sostenibilidad e incidencia en el desarrollo empresarial y social. Para algunos entrevistados la formación a nivel de técnica profesional o de tecnología debe estar referenciada por los intereses directos de las empresas, dicen que este debe ser su fin último y debe ser el criterio de calidad más importante. Expresaron que la razón de ser de un programa de técnica profesional en desarrollo de software, tendría que estar armonizado curricularmente con las herramientas de programación específica y plataformas que se esté utilizando en el momento en las empresas dedicadas al desarrollo de aplicaciones de software.

A las empresas, más que el título profesional les interesa es la especificidad de los conocimientos y las competencias e inclusive, con tal de tener la competencia. Se observa que la titulación puede ser de Ingeniero o de tecnólogo de sistemas, informática o electrónica, por ejemplo. Se nota también que el enunciado del requerimiento directo de tipo técnico es más por la función o responsabilidad específica, que por la titulación académica. Asimismo, se observa que con los requisitos técnicos se suman otros relacionados con competencias transversales o genéricas que deben tener los aspirantes a los cargos.

Las inquietudes que surgen al respecto de la relación entre currículo y empresa, pueden plasmarse con varios interrogantes: ¿Como las instituciones de educación superior están conectadas con las características del empleo en la industria de las TIC? ¿Es necesario complementar la formación profesional con la formación empresarial enfocada en certificaciones, diplomados y especializaciones? ¿Cuál es el sentido de la formación a nivel de maestría o doctorado, sabiendo que no son una atracción inmediata para las empresas?

Una inquietud redundante en los análisis y debates con las fuentes académicas consultadas, es el de la pertinencia expresada en términos de cuáles son las competencias que desde el punto de vista de las empresas deben tener los ingenieros para su desempeño profesional. No cabe duda acerca de la importancia de esta fuente de información, ya que las empresas son el escenario directo donde los profesionales conviven con los proyectos, necesidades urgentes y en general, con las dinámicas productivas y de servicio.

Como consenso importante de los grupos focales, quedaron sugerencias relacionadas con la 
naturaleza de la formación en las facultades de Ingeniería, que deben considerar actualizaciones con el contexto externo a las universidades, para no ir de espaldas a la realidad de la región, el país y el mundo. Con respecto al NISIA, se evidenció que su protagonismo tiene unas dimensiones vigentes dentro del desarrollo de los negocios en las industrias de la información y la comunicación y por eso, dadas las oportunidades presentes y futuras para el resurgimiento económico de las regiones, la educación profesional en esta rama de ingeniería debe ser producto de análisis permanente en los sectores académicos y empresariales.

En otro aspecto, se concluye que, según las fuentes, las empresas requieren de ingenieros comprometidos, competentes y en permanente desarrollo. El compromiso tiene que ver con estar siempre atentos no a sus intereses personales sino a las necesidades de la empresa. Su competencia debe ser integral, tanto desde lo técnico como desde lo personal y el permanente desarrollo implica que demuestre siempre actitudes abiertas para la capacitación, la innovación y el entendimiento de las nuevas tendencias informáticas que vayan surgiendo.

En la encuesta aplicada a ingenieros del sector empresarial, el $73.9 \%$ afirmó que los ingenieros de NISIA deben tener la habilidad para aplicar lenguajes de programación, gestores de bases de datos, frameworks, o middlewares; el 87\% afirmó que deben tener la competencia para liderar procesos de análisis e interpretación de requisitos de soluciones informáticas; el $91.3 \%$ dijo que habilidad para identificar, formular, planear y resolver problemas de sistemas de Información; el $78.3 \%$ dijo que su competencia fundamental es la capacidad para Integración de equipos interdisciplinarios.

Pasando a otro tema, casi todos los entrevistados hicieron hincapié en el alejamiento entre las universidades y el sector empresarial. Pero al mismo tiempo, sin excepción todos coincidieron en que la universidad no debe formar exclusivamente para los intereses de las empresas, pues además de competencias laborales los ingenieros deben formarse para la convivencia, la afectividad, los valores y para ser personas, antes que ingenieros.

Algunos entrevistados coincidieron en afirmar que se debe conservar un equilibrio entre lo que se debe formar como universidad y lo que requiere el sector productivo. Porque si solo se forma para el sector productivo se estaría renunciando a la investigación para crear nuevo conocimiento, ya que hay conocimiento que aún no existen y que por eso el sector productivo no lo utiliza. La generalidad de los entrevistados afirmó que no se puede renunciar a la formación de seres humanos integrales y competentes, solo para dar respuesta a un sector en particular.

\subsection{Competencias, innovación e impacto social}

En los diálogos con decanos, coordinadores de programa, directores de programa y profesores, se plantearon inquietudes sobre las siguientes unidades de análisis relativas al NISIA: aspectos curriculares y pedagógicos, denominación de los programas, dificultades para la matrícula de estudiantes nuevos y la deserción temprana, estrategias para el acercamiento al sector empresarial e Innovación e impacto social.

Se afirmó que los organismos internacionales han pedido a la política pública educativa que se haga énfasis en la tecnología. Sin embargo esto se ha interpretado mal y no se ha entendido la tecnología como el cúmulo de conocimientos ordenados, lógicos y específicos de una técnica, sino como programas académicos o niveles de formación y por esto con los ciclos propedéuticos y con la noción de competencias laborales, se le dio impulso masivo a la creación de programas en el nivel de Tecnología. Lo que quieren decir estos organismos internacionales es que se deben formar para el desarrollo tecnológico, lo cual implica es ingeniería y tecnología informática de alto nivel y no programas técnicos y tecnológicos masificados para la rápida graduación, sin criterios de calidad y alineados con la rápida formación de estudiantes.

Alrededor de estas preocupaciones se afirmó que la formación por competencias en Colombia perdió su norte en la educación superior debido a que las instituciones han intentado incorporar currículos sin la adecuada preparación de aulas y talleres. Entonces aunque hayan currículos por competencias 0 planes de estudio por competencias, las diferencias curriculares no se ven y no hay tal renovación curricular y todo sigue lo mismo en el fondo. La institución y las clases siguen siendo las mismas.

Se dijo que estas dificultades son aún más acentuadas en la educación virtual, pues hay 
programas académicos virtuales que dicen tener currículos por competencias, sin que haya escenarios para prácticas, laboratorios virtuales o procesos con empresas donde los estudiantes puedan practicar efectivamente y donde puedan encontrase con la realidad.

Según expresaron los entrevistados, para ser innovadores los ingenieros deben tener capacidad para presentar solución a problemas, competencias investigativas y pensamiento estratégico y menos funciones operativas.

El proceso de investigación debe estar de la mano de la industria. Se puede hacer mucho más si en las empresas circulan las investigaciones. ¿Cómo hacerlo? Haciendo grupos de investigación vinculando a las empresas. Las empresas no deben únicamente vincularse a los proyectos, sino además, a los grupos de investigación.

Se afirmó que la investigación debe marcar la diferencia entre la formación técnica y la formación profesional. Ya que la investigación es lo que permite la generación e integración de nuevo conocimiento. La investigación en Ingeniería debe ser investigación aplicada, la investigación básica debe ser para las ciencias básicas como la biología - las ciencias sociales, pero en los programas de NISIA debe promoverse la investigación aplicada. Las fuentes consultadas expresaron que para las instituciones de educación superior en Colombia la esperanza de formar para la innovación en tecnología tiene una barrera estructural y es que en el país no se produce tecnología.

Además, se dijo que la investigación debe estar inmersa en el currículo, con unos propósitos que deben trascender hacia ámbitos amplios, identificando situaciones problemáticas y trascendiendo de enfoques objetivos puntuales para productos puntuales, hasta llegar a soluciones estratégicas en convergencia con las realidades sociales.

\section{DISCUSIÓN}

De acuerdo con los datos obtenidos sobre cantidad de estudiantes matriculados y egresados, la inquietud que queda es si en realidad se ha presentado sobreoferta de ingenieros del NISIA con respecto a los demás programas, como a veces se ha afirmado. Las conjeturas acerca de esta sobreoferta de programas del NISIA no son ciertas totalmente, ya que las demandas de tecnología informática en las empresas son crecientes y según lo que revelan los datos del SNIES, en comparación con otros núcleos de conocimiento, el incremento de egresados y el número de programas activos no demuestran diferencias abismales.

Sobre la diversidad de denominaciones, es preocupante la carencia en la identidad gnoseológica del NISIA, pues estructuralmente las diferencias en los planes de estudio y los currículos son estrechas, aunque los programas supuestamente sean distintos. Si se hiciera el ejercicio de un cambio de denominación de algún programa, pocas alteraciones se presentarían entre el plan de estudios actual y el de la nueva denominación, en el caso de tomarse como referencia a programas académicos existentes. Agréguese que el tema de las denominaciones tiene más intereses académicos que empresariales, pues en este último escenario más que especificidad de las titulaciones, lo que interesa es lo relacionado con las competencias profesionales.

En relación con la enseñanza, aunque se reconoce que los éxitos históricos en el desarrollo de la ingeniería justifican la forma catedrática como se ha enseñado, hay también asuntos preocupantes relacionados con la formación integral, como el de la ética, por ejemplo. Sin que se pierda la rigurosidad científica, se requieren propuestas de cambio en la enseñanza y reflexionar sobre la viabilidad del paradigma clásico de enseñanza y la rigidez de los ambientes de aprendizaje y evaluación. Debe tenerse en cuenta que probablemente la ingeniería no se desprestigie por renovar la forma de enseñanza y por estar a tono con las circunstancias y contexto de los estudiantes del Sigo XXI. Debe reconocerse que ellos aprenden de manera distinta a como lo hicieron sus profesores y que además, están hiper-conectados e inmersos en redes y dispositivos tecnológicos que agilizan las comunicaciones y conllevan a mayor facilidad para las relaciones con otros.

Dentro de tal realidad manifiesta, dos enfoques de enseñanza en el NISIA marcan tendencia: uno tradicional, catedrático, basado en la rigurosidad de los procesos de evaluación (propio de las facultades de ingeniería de instituciones reconocidas por su calidad y arraigo) y otro que aboga por flexibilidad en la evaluación, menos 
intensidad en los contenidos, menos afán por la cientificidad y con metodologías contextualizadas con la realidad de los estudiantes (notable en instituciones emergentes, abiertas en soluciones para la inclusión y la cobertura educativa).

Pero aun con estas diferencias, hay también puntos de encuentro. En contextos tradicionales de enseñanza y en los emergentes han surgido las estrategias de Aprendizaje Basado en Problemas y Aprendizaje Basado en Proyectos como alternativas para llenar de significados y motivaciones la formación de los estudiantes en el NISIA. Esto demuestras la preocupación por llevar a los currículos iniciativas metodológicas distintas y por hacer más significativos los procesos de enseñanza y de aprendizaje.

Otro punto de análisis es el de las ciencias básicas. No es raro escuchar opiniones sobre su inconveniencia e inutilidad en los programas de NISIA, haciendo alusión a que los ingenieros de este núcleo no aplican directamente conceptos de cálculo o modelación matemática en sus puestos de trabajo, como si ocurre por ejemplo en la ingeniería civil o la ingeniería mecánica. Pero paradójicamente, tanto los académicos como las fuentes empresariales defendieron los procesos curriculares para fomentar el conocimiento y aptitud matemática. Según esto, se desprende que quienes son antagónicos a la presencia de las ciencias básicas en el NISIA, no entienden que la modulación matemática es esencial para la calidad en los procesos de desarrollo de software, el análisis e interpretación de datos en experimentación con prototipos o en simulaciones para resolver problemas. Podría decirse que ellos conciben la informática como la gestión operativa de dispositivos y no como la automatización estratégica de la información.

Dicho lo anterior, los procesos curriculares de ciencias básicas deben reconocerse desde dos tensiones: la necesidad de crear ambientes significativos flexibles, amigables para los estudiantes, conectados con sus realidades situacionales y asimismo, comprender que es menester el tratamiento riguroso de la modelación matemática y la representación simbólica de la información. Los profesores deben tener claro que aunque sea imperioso llevar a las clases metodologías y recursos para ambientes de aprendizaje centrados en el estudiante, no pueden perder de vista el nivel de logro mínimo de los procesos de evaluación, ni la completitud de los contenidos que deben abordarse. Es decir, las iniciativas de motivación a los estudiantes a partir del facilismo deben evitarse, pues pueden convertirse en pseudo - satisfactores de las necesidades de aprendizaje real [22].

Con respecto a la educación virtual, después de los análisis de los grupos focales quedan muchas inquietudes, especialmente porque hay unos supuestos iniciales que no se sabe hasta donde sean ciertos y que están relacionados con el rol investigativo y autodidacta del estudiante, la suficiencia pedagógica de las aulas virtuales y la responsabilidad de los actores (profesores, estudiantes, directivos) en el desarrollo de las actividades. Sobre estos tres asuntos hay diferencias grandes entre lo que está escrito en los proyectos educativos y lo que verdaderamente ocurre en las aulas virtuales. Incluso, en las instituciones donde se desarrollan programas de NISIA en la modalidad virtual, pareciera que los análisis de los resultados de las investigaciones cuantitativas que se desarrollan, intentaran solapar u ocultar el real sentir de los estudiantes [23].

En consideración del rol investigativo y autodidacta del estudiante, si como se expresó en los hallazgos hay en la modalidad virtual insuficiencias al respecto, entonces ¿Cómo aprender lógica de programación, por ejemplo? e igualmente ¿Cómo aprender ingeniería del software, sabiendo que en este caso es imperativo el discurso amplio del profesor, desde su experiencia y la interacción en las clases alternativas para el modelado? Sobre la suficiencia pedagógica de las aulas virtuales quedan también dudas si se compara con la actividad intensa en las aulas presenciales en procesos de aprendizaje de contenidos como lenguajes de programación 0 matemáticas discretas. En casos como estos, es latente el ímpetu del docente y su inteligencia para crear sobre la marcha ejemplos, contraejemplos y casos que den luces a los estudiantes. El aula virtual puede tener dotaciones tecnológicas para la comunicación en tiempo real, pero su estrechez es manifiesta para eventos como los anteriores. Pero además, queda también pendiente la claridad sobre los laboratorios y las practicas ¿Sera que todas puedan ser simuladas con software o ambientes de hipermedia?

A lo anterior agréguese que aún permanece un ambiente de dudas sobre esta modalidad 
educativa, especialmente cuando no hay respuestas sobre la alineación de la enseñanza en el ambiente virtual con características formativas de los ingenieros de este núcleo, como representación de la información, pensamiento secuencial, enfoque sistémico, versatilidad analítica o aplicación de artefactos físicos.

Con relación a la responsabilidad de los actores, llama la atención que sean ellos mismos (estudiantes, egresados y profesores de la modalidad virtual del NISIA), quienes esgrimen argumentos que cuestionan la autenticidad en las actividades formativas. Asimismo, son ellos quienes plantean descontentos con la ética en las decisiones de directivos y con las actuaciones de compañeros y colegas, en algunos casos.

Pasando al tema de la educación técnica profesional y tecnológica, no cabe duda que su presencia y su importancia para las empresas tienen plena vigencia. Esto en contraposición al sentir observado tradicionalmente en las elites académicas universitarias que han mirado con cierto estigma a los técnicos y tecnólogos en el NISIA. Ellos son esenciales para la productividad de las empresas y allí son valorados, pero en las universidades los ven como si fueran profesionales de segunda clase. A esto súmese que políticas públicas como los ciclos propedéuticos han impulsado la estadía de paso o temporal de los estudiantes en los niveles de técnica y tecnología, pues se motiva que el verdadero fin es llegar a ser ingenieros y no el aporte a las empresas desde sus competencias. .

Una discusión continua es la que gira alrededor de la relación entre currículo y sector empresarial, evidenciándose dos consideraciones. La primera es que la finitud de los planes de estudio pueden resultar insuficientes para abarcar el mayor número de tecnologías que reclamen las empresas, deduciéndose con esto en mente, que la academia en ingeniería debe preparar a los estudiantes con unos conceptos básicos y generales, pero actualizados, de manera que fácilmente puedan asumir el aprendizaje de cualquier tecnología emergente. Para esta preparación es indiscutible que deben plasmarse ambientes de aprendizajes pertinentes y contenidos curriculares que comulguen tanto con intereses científicos y de fundamentación, como con las tendencias informáticas globales. La segunda está relacionada con la extensión académica y expresa que, casi obligadamente los estudiantes y egresados deben asumir la responsabilidad de formación en temas informáticos empresariales, sea a nivel de certificaciones, cursos, diplomados, seminarios o demás. Agréguese que el autoaprendizaje hace parte del ciclo de vida profesional de los programas del NISIA y de esto debe estar convencida la comunidad académica en las instituciones de educación superior. La tercera apunta a que debe reconocerse la educación empresarial (capacitación o cualificación) como un componente importante de la profesionalización, siendo por esto responsabilidad de las empresas y los empleados el desarrollo de eventos de formación específica sobre las tecnologías directamente relacionadas con la productividad y el negocio.

Son evidentes las paradojas de intereses entre lo global y lo regional. Dentro de los hallazgos se encontró cierto consensos acerca de la necesidad de crear procesos curriculares para formar ingenieros pensantes de manera global, es decir, conocedores de las tensiones locales y extranjeras y capacitados para adaptarse a colegas que llegan al país para desarrollar proyectos y al mismo tiempo, para ir a otros países a integrarse calificadamente en equipos especializados en tecnología. Pero de igual manera, no debe perderse de vista el interés en la región y en sus condiciones reales, donde muchas veces se hace necesario educar a los ingenieros para para sus contextos locales. En este sentido no debe perderse de vista que la realidad regional muestra que el objetivo empresarial tiene menos interés en desarrollar tecnología, y más en adaptarla y aplicarla en soluciones concretas. Podría decirse que paradojas así planteadas ponen en dudas procesos curriculares enfocados directamente y solo en lo que ocurre o puede ocurrir en la globalidad.

Pasando al tema de los propósitos formativos, debe considerarse que las competencias de los ingenieros del NISIA relacionadas con el soporte y la gestión de informática e infraestructura, se adquieren más desde la experiencia y la capacitación empresarial específica, que desde la formación en las universidades. Indiscutiblemente, una de las funciones de ellos están relacionada con la planeación, implantación y evaluación de infraestructura de informática, y siendo esta función un asunto clave en las organizaciones, desde ahí se perfila una buena oportunidad para el desarrollo profesional, Sin embargo, estas son competencias 
que difícilmente pueden delegarse exclusivamente a los currículos de las instituciones. Aspectos como estos son los que abren reflexiones sobre la identidad de los ingenieros de NISIA [24], [25], [26].

Un asunto de fondo es el de la conexión entre el currículo y las empresas. En el concepto de decanos, profesores y directores de programa, no es adecuado que un programa académico este diseñado totalmente de acuerdo con los intereses de las empresas, ya que el estudiante requiere además de competencias laborales, oportunidades de formación integral. Además, hay heterogeneidad en el sector productivo, porque por ejemplo, en el sector de tecnologías de la información (TI), hay industrias desarrollando software con herramientas muy específicas y si las instituciones de educación superior se encaminan concretamente en esas herramientas, se estaría descuidando las necesidades de otros tipos de empresas que requerirían de otro tipo de herramienta. De esta forma ninguna institución de educación superior estaría en capacidad de responder a todas las necesidades de cada empresa. Por eso la formación exclusiva para el sector empresarial no es la adecuada. Además hay una particularidad: el sector TI es demasiado cambiante, por eso hay que capacitar a los futuros egresados para que sean capaces de aprender rápidamente y de adaptarse a nuevas tecnologías y paradigmas. $\mathrm{Si}$ las instituciones se enfocan en una sola herramienta, el estudiante perdería la oportunidad de construir su propio conocimiento y su habilidad para adaptarse a una nueva tecnología.

\section{CONCLUSIONES}

1) Se evidencia cierta crisis en la identidad gnoseológica del NISIA, ya que, aunque en la oferta académica se vislumbran distintas denominaciones de los programas académicos las diferencias no son evidentes en los aspectos curriculares, Sobre la estructura de los planes de estudio, la tendencia observada es la composición de unas áreas obligatorias y otra optativa, con créditos de libre elección en unos casos y obligatorios en otros. Además, la tendencia es hacer más livianos los planes de estudios, menos densos y más especializados.
2) En relación con las ciencias básicas, su presencia en los planes de estudio de los programas de NISIA es generalizada y su importancia reconocida, tantos en el sector académico como en el empresarial. Pero tal vez por desconocimiento de lo fundamental de la informática, son comunes los debates y las posturas acerca de su conveniencia y utilidad.

3) Sobre la educación virtual en el caso del NISIA, aunque su desarrollo ha sido afanoso y muchas expectativas se han trazado, no hay certezas sobre su calidad y sobre la ética en los procesos formativos, lo que impide que se pueda colocar a un mismo nivel de la educación presencial.

4) Con respecto a la educación técnica profesional y tecnológica, se concluye que su importancia es latente, pero no como estadios de paso para la cadena formativa que culmina con el grado de ingeniero (ciclos propedéuticos). Lo ideal es que técnicos y tecnólogos tengan la suficiente preparación para aportar a la competitividad empresarial y que tengan los suficientes reconocimientos y estímulos tanto en las universidades como en las empresas. La tendencia es formar técnicos y tecnólogos con criterios de calidad y pertinencia, en conexión con las empresas, de manera que los egresados encuentren en este nivel significado a su proyecto de vida.

5) En el tema de las expectativas de las empresas, puede decirse que las competencias transversales y el perfil personal sobresalen, en comparación con otros criterios de selección y vinculación de profesionales. En los asuntos técnicos, para las empresas más que la titulación lo importante son las competencias, conocimientos y experiencias sobre la aplicación de la tecnología informática para la productividad y por esto, muy buena valoración se le dan a las certificaciones empresariales. Cabe mencionar también, que en general la consideración es que las instituciones de educación superior no están formando, en el aspecto técnico a los ingenieros que se requieren en las empresas. 
6) Las percepciones de los académicos sobre el currículo y la situación actual de los programas de NISIA, son variadas y a veces hasta contradictorias. No obstante hay un consenso: los programas de NISIA deben formar integralmente a los ingenieros y no exclusivamente para los intereses y necesidades técnicas de las empresas. Se destaca también que sus percepciones sobre las causas de la crisis en la matrícula de estudiantes nuevos y de la deserción temprana, apuntan casi que de manera generalizada, a tres factores: el temor por las ciencias básicas que se infunde desde las instituciones de educación básica y media; la propensión de los jóvenes del siglo XXI al estudio de carreras cuyas metodologías están más enfocadas en la interacción personal y la flexibilidad dentro de las aulas, que en el estudio concentrado, estricto, disciplinado que por lo general caracteriza a la ingeniería.

\section{AGRADECIMIENTOS}

A los decanos, directores de programa y profesores de ingeniería de sistemas, informática o afines que colaboraron con el proyecto de investigación.

\section{REFERENCIAS BIBLIOGRÁFICAS}

[1] Asociación Colombiana de Facultades de Ingeniería (ACOFI), "Marco de Fundamentación Conceptual. Especificaciones de Prueba ECAES de Ingeniería de Sistemas. Versión 6 ", Bogotá, 2005.

[2] E. Parra, "Las ciencias básicas en ingeniería de sistemas: justificaciones gnoseológicas desde los objetos de estudio y de conocimiento", Revista Educación en Ingeniería, no 10, pp. 74-84, 2010.

[3] T. Marjoran \& Y. Zhong, " What engineering is, what engineers do?" In: Engineering: Issues Challenges and Opportunities for Development, Paris: UNESCO Publishing.2010.

[4] J. A. Rodríguez, "Códigos de comunicación y el proceso enseñanza-aprendizaje en la formación del ingeniero", Paradigmas, vol.3, no 1, pp. 145-153, 2018.
[5] Instituto Colombiano para Fomento a la Educación Superior (ICFES) y Asociación Colombiana de Facultades de Ingeniería (ACOFI), "Actualización y modernización del currículo de ingeniería de sistemas", Bogotá, 1997.

[6] J. Villalobos, "Ingeniería de sistemas: paradojas de una crisis", En: Primer Encuentro Nacional de Ingeniería de Sistemas (Colombia 2010). Bogotá: Redis, p. 41, 2011.

[7] P. Rengifo, "La ingeniería de sistemas: una mirada desde la Fundación Universitaria Konrad Lorenz (FUKL)", En: Primer Encuentro Nacional de Ingeniería de Sistemas (Colombia 2010). Bogotá: Redis, p. 67, 2011.

[8] W.R. Avedaño y A. Parada-Trujillo, "El currículo en la sociedad del conocimiento cognitiva", Educación y educadores, vol. 16, no. 1, pp. 159174, 2013.

[9] G. lafrancesco, "Nuevos fundamentos para la transformación curricular: a propósito de los estándares", Bogotá, Cooperativa Editorial Magisterio, 2005.

[10] E. Galeano, “ Diseño de proyectos de investigación cualitativa", Medellín, Fondo Editorial Universidad EAFIT. p. 19, 2004.

[11] R. Sampieri, C. Collado, Carlos y P. Lucio, "Metodología de la Investigación", México D.F., McGraw-Hill, P. 10, 2003.

[12] M. Cajas, "Relación Universidad-Estado Empresa", En: Tercer Encuentro Nacional de Ingeniería de Sistemas (Colombia 2012), Medellín, Redis, p.23, 2013,

[13] R. Martínez, "Universidad y empresa, una alianza exitosa", En: Tercer Encuentro Nacional de Ingeniería de Sistemas (Colombia 2012). Medellín, Redis, p.49, 2013.

[14] L. Suárez, "Lina. Ingeniería de Sistemas: ¿Formación Técnica, Tecnológica o Profesional?”, En: Cuarto Encuentro Nacional de Ingeniería de Sistemas (Colombia 2013). Bogotá, Redis, p.23, 2014.

[15] R. Sotaquirá, "Docencia + investigación + creación: retos del docente universitario en ingeniería informática", En: Quinto Encuentro 
Nacional de Ingeniería de Sistemas (Colombia 2014). Bogotá: Redis, p. 29, 2015.

[16] D. Gómez, "La formación para el Ingeniero de Sistemas comprometido con Colombia", En: Sexto Encuentro Nacional de Ingeniería de Sistemas (Colombia 2015), Bogotá: Redis, p.12, 2016.

[17] M. Sepúlveda, "El Ingeniero de Sistemas, profesional de retos", En: Sexto Encuentro Nacional de Ingeniería de Sistemas (Colombia 2015). Bogotá: Redis, p.24, 2016.

[18] C. Paredes, "Aprendizaje basado en problemas (ABP): una estrategia de enseñanza de la educación ambiental, en estudiantes de un Liceo Municipal de Cañete", Revista Electrónica Educare, vol. 20, no. 1, 2016.

[19] J. Zamora y O. Morato, "Aprendizaje basado en problemas como recurso pedagógico en el cambio de la conducta sedentaria en jóvenes universitarios", Revista chilena de salud pública, vol. 20, no 2, pp. 113-121, 2016.

[20] J. E. Parra, M.J., Amariles y C.A. Castro, "Aprendizaje basado en problemas en el camino a la innovación en ingeniería", Ingenierías USBMed, vol 7, no. 2, pp 96-103, 2016.

[21] J.E. Parra, C.A. Castro y M.J. Amariles (2014), "Casos de éxito de la aplicación de la metodología de aprendizaje basado en problemas ABP ", IngEam, no 1, pp. 15-22, 2014.

[22] A. Elizalde, "Desarrollo a escala humana: conceptos y experiencias, Interações (Campo Grande), vol1, no 10, pp. 51-62, 2016.

[23] L. Montoya, M. Sepúlveda, E. Pulgarín y J. Botero, "Estudio de percepción de los estudiantes del programa de ingeniería de sistemas hacia la modalidad virtual y distancia sobre la enseñanza y aprendizaje en plataforma virtual Remington 2015 2016", En: Encuentro Internacional de Educación en Ingeniería ACOFI 2017 (Colombia, 2017). Bogotá: ACOFI, 2017.

[24] L.C. Díaz, "Reflexiones asociadas con la identidad del ingeniero de sistemas. En: Segundo Encuentro Nacional de Ingeniería de Sistemas (Colombia 2011). Bogotá: Redis, p.29, 2012.
[25] J. Martínez, "Hacia la identidad del ingeniero de sistemas. En: Segundo Encuentro Nacional de Ingeniería de Sistemas (Colombia 2011)", Bogotá: Redis, p. 31, 2012.

[26] L. Molina, "Identidad del ingeniero de sistemas", En: Segundo Encuentro Nacional de Ingeniería de Sistemas (Colombia 2011), Bogotá, Redis, p. 35, 2012. 